Pedagogía y Saberes No. 50 Universidad Pedagógica Nacional

Facultad de Educación. 2019.pp. 63-74

\title{
¿Cuál es la tarea de la educación? Despertando el deseo de querer existir en el mundo de una manera adulta
}

Traducción

\section{What is the Educational Task? Arousing the Desire for Wanting to Exist in the World in a Grown-up Way Qual é o dever da educação? Despertando o desejo de querer existir no mundo de uma maneira adulta}

Gert Biesta*

Traducido por: Carlos Ernesto Noguera-Ramírez**

\section{Para citar este artículo}

Biesta, G. (2019). ¿Cuál es la tarea de la educación? Despertando el deseo de querer existir en el mundo de una manera adulta (Trad. C. Noguera-Ramírez). Pedagogía y Saberes, 50, 63-74.

* Profesor e investigador del Departamento de Educación y director de investigación de Brunel University London. Su trabajo se centra en la teoría y la filosofía de la educación, así como en el análisis de la política educativa con particular interés en el análisis de cuestiones relacionadas con la democracia y la democratización de la educación.

Correo electrónico: gertbiesta@gmail.com

Código oRCID: orcid.org/0000-0001-8530-7105

** Profesor de la Facultad de Educación de la Universidad Pedagógica Nacional, Departamento de Psicopedagogía; doctor en Educación y magíster en Historia. Investigador del Grupo de Historia de la Práctica Pedagógica-GHPP, del Grupo de Estudos e Pesquisas em Currículo e Pós-modernidade-gepcpós da UfRGS y del Grupo de Estudo e Pesquisa em InclusãoGEPI da unisinos.

Correo electrónico: cnoguera@pedagogica.edu.co Código oRciD: orcid.org/0000-0001-9848-0724 


\title{
Resumen
}

Las discusiones sobre educación a menudo están enfocadas en los propósitos que deberían lograrse o en los resultados que deberían producirse. En este artículo abordamos la cuestión del propósito de la educación de forma diferente, al preguntarnos en qué debería enfocarse el trabajo de los profesores y demás educadores. La respuesta que proponemos es que el objetivo de este trabajo es despertar en los niños y en los jóvenes el deseo de querer existir en este mundo, de la forma que denominamos como "adulta". Explicamos nuestra idea de "crecimiento", sobre la que argumentaremos que no deberíamos pensar en ella como el resultado de una trayectoria de desarrollo, sino como una forma en la que los seres humanos pueden tratar de existir en y con el mundo. Esto sugiere que es importante, en el trabajo educativo, interrumpir las formas en las que los niños y los jóvenes están completamente enfocados en sí mismos, llevándolos así al mundo en que solo se puede dar su existencia como sujetos adultos. La educación enfocada en eso tiene la tarea de ofrecer tiempo, espacio y formas curriculares a través de las cuales los niños y los jóvenes puedan trabajar "en" su crecimiento. Adicionalmente, la educación debe proveer sustento para que niños y jóvenes puedan recibir apoyo y nutrirse en su tarea de intentar existir de forma adulta.

\section{Palabras clave}

educación; enseñanza; crecimiento; egocentrismo; interrupción; currículo

\begin{abstract}
Discussions about education often focus on the purposes that should be achieved or the outcomes that should be produced. In this paper I approach the question of the purpose of education differently, by asking what the work of teachers and other educators should focus. The answer I propose is that this work is about arousing the desire in children and young people for wanting to exist in the world in what I refer to as a "grown-up" way. I explain my use of the idea of "grown-up-ness," where I argue that we should not think of this as the outcome of a developmental trajectory but as a way in which human beings can try to exist in and with the world. This suggests, as I explain, that central to the work of education is the interruption of ways in which children and young people are entirely self-centred and self-focused, thus turning them towards the world in which their existence as grown-up subjects can only take place. Education that is focused on this has a task to provide time, space and curricular forms through which children and young people can work "on" their grown-up-ness. And education needs to provide sustenance so that children and young people can be supported and nourished in their task of trying to exist in a grown-up way.
\end{abstract}

\section{Keywords}

education; teaching; grown-up-ness; egocentrism; interruption; curriculum

\section{Resumo}

As discussões sobre educação estão focadas, com frequência, nos propósitos que deveriam ser conquistados ou nos resultados que deveriam ser produzidos. Neste artigo abordamos a questão do propósito da educação de forma diferente, perguntando em que deveria estar focado o trabalho dos professores e outros educadores. A resposta que propomos é que o objetivo deste trabalho é despertar o desejo, nas crianças e jovens, de querer existir nesse mundo, da forma que denominamos como "adulta". Explicamos nossa ideia de "crescimento", sobre a qual argumentamos que não deveríamos pensar nela como o resultado de uma trajetória de desenvolvimento, mas como em uma forma na que os seres humanos podem tentar existir em e com o mundo. Isso sugere que é importante, no trabalho educacional, a interrupção de formas nas que as crianças e os jovens estão inteiramente focados em si mesmos, levando-os assim para o mundo em que sua existência como sujeitos adultos só pode acontecer. A educação focalizada nisso tem a tarefa de fornecer tempo, espaço e formas curriculares através das quais crianças e jovens possam trabalhar "em" seu crescimento. Adicionalmente, a educação precisa prover sustento para que crianças e jovens possam ser apoiados e nutridos em sua tarefa de tentar existir de forma adulta.

\section{Palavras-chave}

educação; ensino; crescimento; egocentrismo; interrupção; currículo 
$\mathrm{E}$ n este artículo exploro, de forma sencilla y en un sentido básico, la pregunta "¿Cuál es la tarea de la educación?"

Soy conciente de que la redacción de esta pregunta no es la ideal, sobre todo en lo que respecta a la palabra "tarea". En las lenguas germánicas hay muchas palabras más precisas e interesantes para referirse a lo que busco. En alemán existen las palabras Aufgabe y Auftrag, que se asemejan mucho a las palabras holandesas opgave y opdracht. Lo que estas palabras tratan de insinuar son cosas que necesitamos hacer, que están ahí para que nosotros las realicemos cuando nos encontramos en una determinada posición o encargo, como el encargo de maestro o educador. Se trata menos de una tarea que debe ser realizada o un trabajo por hacer y más de una responsabilidad en la que nos encontramos. Curiosamente, las palabras Gabe y gave en realidad significan "dádiva", por lo que Aufgabe y opgave se refieren a una tarea que se nos da, una tarea que viene con el trabajo o, por así decirlo, la responsabilidad que viene con el encargo. Auftrag y opdracht incluyen las palabras tragen y dragen, que significa "cargar", y eso es lo que la tarea que nos ha sido encomendada nos pide. Que carguemos con la tarea. Lo que busco expresar a través de la pregunta por la tarea educativa, por tanto, es que la educación no es solo lo que queremos que sea, sino que viene con un particular Aufgabe, una responsabilidad particular, e incluso podríamos decir que un imperativo en particular.

La respuesta que sugiero en este artículo es que la tarea educativa consiste en hacer posible la existencia adulta de otro ser humano en y con el mundo. 0 , de manera aún más precisa: la tarea educativa consiste en despertar el deseo en otro ser humano por querer existir en y con el mundo de una manera adulta, es decir, como sujeto. Hay por lo menos dos aspectos en esta respuesta que necesitan una mayor exploración. Una de ellas es la idea "adultez" y la otra el uso de la palabra "existencia". Para empezar con esta última: usar la palabra "existencia" significa que deseo enfocarme en la manera en que los seres humanos existen, es decir, cómo son y no quiénes son. Si esta última es la cuestión de la identidad, la primera es la cuestión de la subjetividad o, en términos un poco más precisos: es la cuestión de la subjetividad o de la "condición" humana de ser sujeto. Ambas cuestiones - la cuestión de quién soy y la cuestión de cómo soy- son, por supuesto, preguntas legítimas en el contexto de la educación. Pero son preguntas muy diferentes y es importante no confundirlas, ni a nivel de concep- tos - los conceptos de "identidad" y "subjetividad" [subject-ness] no son intercambiables- ni en el nivel de lo que estos conceptos buscan expresar.

Como discutiré con más detalle a continuación, me aproximo a la "adultez" - ciertamente una palabra un poco incómoda - no como una etapa de desarrollo o el resultado de una trayectoria de desarrollo, sino en términos existenciales, es decir, como una "cualidad" particular o modo de existir. Lo que distingue a una forma adulta de una manera no adulta de existencia es que la forma adulta reconoce la alteridad y la integridad de qué y quién es el otro, mientras que en la manera no adulta esto no está "en el radar". La forma adulta reconoce, en otras palabras, que el mundo de "ahí afuera" está de hecho "ahí afuera", y no es ni un mundo de nuestra propia fabricación, ni un mundo que solo está a nuestra disposición, es decir, un mundo con el que podemos hacer lo que queremos. "El mundo" se refiere aquí tanto al natural cuanto al mundo social, así como al mundo de las cosas y al mundo de los seres. Se refiere, más concretamente, tanto a nuestro planeta y todo lo que contiene, como a los otros seres humanos que nos encontramos en este planeta. Se refiere, con una palabra interesante propuesta por Alfonso Lingis (1994, p. 123), tanto a la tierra cuanto a los "terrícolas" que la habitan. Reconocer la alteridad y la integridad de este mundo no ha de entenderse como un acto de generosidad de mi parte de dejar qué y quién existir. En otras palabras, no es mi decisión dejar que el mundo exista o no; más bien, es mi decisión de otorgar, o no, a la alteridad e integridad del mundo un lugar en mi vida.

¿Cuál es la justificación para sugerir que la tarea educativa es hacer posible la existencia adulta de otro ser humano en y con el mundo? En un sentido absoluto no existe ninguna justificación para esto y, a este respecto, la sugerencia carece, literalmente, de fundamento. Sin embargo, esto no impide que esta sugerencia pueda ser significativa, sobre todo si se compara con otras alternativas acerca de cuál podría ser la tarea educativa. Un punto a destacar aquí es que, en realidad, solamente en el mundo podemos existir verdaderamente, ya que cuando nos alejemos del mundo terminamos existiendo solo con y para nosotros mismos - cosa que es una forma bastante pobre y egocéntrica de existir, si es que eso es existir-. Existir en y con el mundo plantea siempre la cuestión de la relación entre mi existencia y la existencia del mundo. Y aquí de nuevo, al menos como punto de partida, podemos decir que existir en y con el mundo sin dejar espacio para lo que existe 
allí también, realmente, no es existir en el mundo. El reto, por lo tanto, es existir en el mundo sin considerarnos a nosotros mismos como el centro, el origen o el fundamento del mundo - que es exactamente la manera en que Philippe Meirieu describe el "estudiante sujeto" (élève-sujet), es decir, como aquel que es capaz de vivir en el mundo, sin ocupar el centro del mundo ${ }^{1}$ (Meirieu, 2007, p. 96).

Pero tal vez la pregunta más difícil es por qué debemos pensar en esto como un asunto educativo y no como algo que cada uno de nosotros debe descifrar en su propia vida. En otras palabras, ¿por qué debemos siquiera considerar la sugerencia de que sería la tarea - y por lo tanto la responsabilidad y tal vez incluso el deber - de que un ser humano haga posible la existencia adulta de otro ser humano? Podríamos responder a esta pregunta haciendo referencia al hecho de que esto parece ser lo que los educadores siempre han estado haciendo, que es clave para lo que significa ser un padre y para lo que significa ser un maestro, y que lo que estoy tratando de hacer es simplemente explorar lo que ello podría significar en nuestros tiempos. También podríamos decir que la ambición de hacer posible la existencia adulta de otro ser humano expresa un interés por la libertad y, más específicamente, un interés por la libertad del otro, y que es clave para lo que la educación debe ser (Biesta y Säfström, 2011). Creo que así es que podemos articular el interés educativo $y$, por lo tanto, la tarea educativa; sin embargo, no creo que esto equivalga, automáticamente, a una justificación de la misma. Después de todo, la promesa de la liberación con demasiada frecuencia se ha convertido en otro ejercicio de poder (véase, por ejemplo, Spivak 1988; véase también Biesta 2010a y, para una discusión más amplia, Andreotti, 2011), lo que significa que en estos asuntos hay que proceder con cuidado y no con demasiadas pretensiones.

Presentaré mis reflexiones sobre la tarea educativa en cinco breves pasos, de una parte, en relación con algunas ideas que he presentado con más detalle en publicaciones anteriores, y de otra, destacando con más precisión las nociones de "existencia" y "adultez". Primero, revisaré la noción de subjetividad [subjectness] y trataré de precisar lo que significa existir como sujeto. Discutiré este asunto de manera más detallada con el argumento de que las cuestiones existenciales son, en última instancia, asuntos personales antes que cuestiones teóricas. Voy a explicar esta distinción e indicaré lo que significa la cuestión de ser-sujeto y, más concretamente, la idea de singularidad. Desde

1 En francés: "Un élève-sujet est capaces de vivir dans le monde sans occuper le centre du monde". allí me referiré a la cuestión de lo que significa existir en el mundo, una pregunta que trataré de responder poniendo de relieve lo que significa no estar en el mundo. Esto me permitirá decir algo más acerca de la distinción entre formas adultas y no adultas de estar en el mundo y la importancia de la distinción entre lo deseado y lo deseable. En quinto y último lugar, reflexionaré sobre el "trabajo" educativo que podría contribuir a hacer posible la existencia adulta de otro ser humano en y con el mundo. Terminaré el artículo con una breve reflexión sobre el papel del poder y la autoridad en las relaciones educativas y lo que esto significa para la enseñanza y el maestro.

\section{E1 sujeto está sujeto}

Teniendo en cuenta que he dicho que hablar de la subjetividad [subject-ness] del ser humano no es hablar de su identidad, la primera pregunta es qué significa ser un sujeto. Podemos responder a esta pregunta de dos maneras: mirando hacia el sujeto en sí para luego tratar de averiguar lo que el sujeto es o mirando más allá del sujeto y luego preguntar qué significa para el sujeto existir. Aquí opto por la segunda opción que se inspira en la frase de Sartre de que "la existencia precede a la esencia", es decir, que en primer lugar existimos, que nos "encontramos" a nosotros mismos en la existencia, y que cualquier respuesta a la pregunta sobre quienes somos viene después. ${ }^{2}$ Aunque los intentos de responder a la pregunta sobre lo que es un sujeto no son necesariamente sin sentido, se hacen, en cierto modo, siempre demasiado tarde en relación con nuestra propia existencia. Esto significa que si bien pueden ayudar a clarificar las dimensiones de la condición humana, no son capaces de fundamentarla. Si, inspirándonos en Heidegger, tomamos la idea de la existencia en un sentido literal, podemos empezar a ver un aspecto de la existencia del sujeto, a saber, que existir como sujeto no quiere decir ser uno mismo - ser idénticos a uno mismo- sino más bien estar "fuera" de sí mismo, esto es, de alguna manera "sobre-salir" ("ek-sistir") en el mundo y ser "arrojado" en él.

La idea principal que deseo destacar acerca de la existencia del sujeto y de nuestra existencia como sujetos es que, en gran medida, nuestra subjetividad [subject-ness] no está en nuestras propias manos, o mejor dicho, no está en nuestras manos para nada. El autor que he encontrado más útil para dar sentido

2 Sartre escribe en El existencialismo es un humanismo (Sartre, 2007/1946) que "el hombre en primer lugar existe, se encuentra a sí mismo, resurge en el mundo -y después se define a sí mismo" (p. 22). 
a este aspecto de nuestra subjetividad es Hannah Arendt, en particular sus ideas sobre la acción (que, en la obra de Arendt, es un término técnico con una definición muy precisa). Acción - que para Arendt (1958) es una de las tres modalidades de la vida activa, la vita activa - primero que todo significa tomar la iniciativa, es decir, empezar algo. A diferencia de muchos filósofos que hacen hincapié en la mortalidad del ser humano, Arendt (1958) mira hacia la dirección opuesta, es decir, hacia la capacidad del ser humano de ser un principio y un principiante. Arendt compara la acción al hecho del nacimiento, ya que con cada nacimiento algo "único y nuevo" entra en el mundo (Arendt, 1958, p. 178). Pero no es solo en el momento del nacimiento que esto sucede. A través de nuestras "palabras y hechos" continuamente traemos nuevos comienzos al mundo.

El principio es, sin embargo, solo la mitad de lo que es la acción, porque el hecho de que nuestros comienzos tengan alguna consecuencia, que "vengan al mundo" (Biesta 2006), depende completamente de cómo otros tomarán nuestros comienzos -y "tomar" debe ser entendido en el sentido más amplio posible, incluyendo la respuesta a tales comienzos, repitiendo tales comienzos, tomando tales comienzos como una señal para nuevas iniciativas, y así sucesivamente- Es por eso que Arendt (1958) escribe que el "agente" no es un autor o un productor, sino un sujeto en el doble sentido de la palabra, es decir, como el que comenzó una acción y el que sufre y está, literalmente, sujeto a sus consecuencias. Lo más importante de todo esto es que nuestra "capacidad" de acción - que en este sentido no es precisamente una capacidad que tenemos o poseemos-depende decisivamente de las formas en que otros toman nuestros comienzos. En este sentido podemos decir que nuestra subjetividad [subjec-ness] no está en nuestras propias manos, lo que podríamos resumir, como lo hace Simon Critchely (1999 p. 63), diciendo que "el sujeto está sujeto".

Aunque el interés de otros por nuestras iniciativas haya frustrado nuestros comienzos, Arendt (1958) enfatiza una y otra vez que la "imposibilidad de seguir siendo maestros únicos de lo que hacemos" es la condición, la única condición bajo la cual nuestros comienzos pueden llegar a ser reales, es decir, pueden venir al mundo (p. 244). Por lo tanto, también es la única condición bajo la cual podemos entrar en el mundo, o sea, podemos existir como sujetos. Si bien puede ser tentador querer controlar las formas en que otros toman nuestros comienzos, el problema es que tan pronto como lo hacemos, empezamos a privar a otros de sus oportunidades de acción, de sus oportunidades de comenzar y de existir como sujetos. En ese caso estaríamos en un mundo donde una persona puede actuar - puede ser un sujetoy todos los demás son solo seguidores, y por ende, un objeto de aquel quien es sujeto. Arendt concluye que la acción nunca es posible aisladamente, lo que también significa que nunca podremos existir como sujetos aislados. Arendt (1958) incluso argumenta que "aislarse es privarse de la capacidad de actuar" (p. 188). Esto, a su vez, la lleva a la afirmación simple pero profunda de que "pluralidad es la condición de la acción humana" (p. 8), es decir, que solo es bajo la condición de pluralidad que la acción para todos -y por ende la subjetividad [subject-ness] para todos- es posible. Es importante no leer esto como una declaración empírica, sino como el "núcleo" normativo del trabajo de Arendt, que está explícitamente comprometido con un mundo en el que todos tienen la oportunidad de actuar y de existir como sujetos (véase también Biesta, 2010b).

\section{Singularidad como irremplazabilidad}

Mientras que Arendt nos ayuda a dar sentido a la idea de que nuestra subjetividad no está en nuestras manos, sino que en gran medida depende de lo que otros hacen con nuestras iniciativas, hay aún dos limitaciones para su aproximación, limitaciones que en cierto sentido están conectadas. Una limitación es que Arendt nos proporciona una teoría acerca de la subjetividad humana y, por lo tanto, se aproxima a la cuestión de la subjetividad desde lo que podríamos llamar una "perspectiva en tercera persona". Si bien sus ideas son esclarecedoras, tratan de dar una descripción de la condición del ser-sujeto desde el "exterior", por así decirlo, sino más bien desde el punto de vista de la existencia del sujeto mismo - lo cual podría hacer referencia a una perspectiva "en primera persona"- La segunda limitación es que Arendt proporciona una explicación general de la condición de la subjetividad humana en lugar de una explicación de cada sujeto humano en su singularidad. Para decirlo más crudamente: mientras que Arendt nos aproxima a la comprensión de lo que significa para los sujetos existir, no nos da un argumento de por qué podría ser importante que cada sujeto humano individual exista. Estas observaciones pueden sonar vagas y extrañas cuando se hacen de esta manera abstracta, pero son precisamente lo que está en juego en la forma en que Emmanuel Levinas se aproxima a la cuestión de la subjetividad humana que intenta explicar, no en la forma de una teoría, sino desde la perspectiva de la primera persona. Aquí la subjetividad aparece como algo que tengo que 
descifrar y que nadie más puede descifrar por mí, y tampoco es algo que puedo descifrar para ninguna otra persona. Y el término clave en la explicación de Levinas es "singularidad".

Sin embargo, la singularidad es un término complicado, ya que en una primera impresión podríamos entenderlo desde la perspectiva de la tercera persona, es decir, como la cuestión relativa a las características y capacidades que hacen que cada uno de nosotros sea diferente a todos los demás. Esta primera impresión sugiere la idea de singularidad-como-diferencia que nos llevaría inmediatamente a cuestiones de identidad e identificación y a una perspectiva externa sobre la singularidad - donde, desde un punto de vista abstracto, podríamos aclarar cómo cada ser humano es en cierto modo diferente a cualquier otro. Según mi lectura de su trabajo, Levinas apunta hacia una pregunta diferente sobre la singularidad, que no es la pregunta "¿Qué me hace único?"- la pregunta acerca de lo que tengo que me hace diferente de todos los demás - sino la pregunta "¿Cuándo importa que yo sea yo?"- Esta última pregunta no indaga, precisamente, acerca de todo lo que tengo o poseo que me distinga de los demás, sino que busca situaciones, eventos existenciales donde mi singularidad está "en juego"y donde yo, por lo tanto, estoy en juego. Las situaciones que Levinas tiene en mente son aquellas donde alguien me hace un llamado de forma tal que el llamado está dirigido a mí y a ningún otro. Se trata de situaciones donde el llamado viene hacia mí y solamente yo puedo responder. Son, en otras palabras, situaciones en las que encontramos una responsabilidad, que es la razón por la que Levinas sugiere que la responsabilidad es "la estructura esencial, primaria y fundamental de la subjetividad" (Levinas, 1985, p. 95).

Alfonso Lingis (1994) ofrece el ejemplo útil de un caso en el que una amiga que se está muriendo le pregunta si puede verlo. Tal pregunta, Lingis argumenta, es una pregunta que solo fue dirigida a usted, pues a la amiga no le interesa ver a alguien, quiere verlo a usted y a nadie más. Esta es una pregunta, por tanto, que, literalmente, lo singulariza. Es una pregunta que grava con una responsabilidad. Es para que usted pueda asumir esta responsabilidad o rechazarla. Cuando Zygmunt Bauman resume las ideas de Levinas escribiendo que para Levinas la responsabilidad es "la primera realidad del yo" (Bauman, 1993, p. 13), capta muy bien lo que estamos discutiendo aquí, ya que podríamos decir que es solo en encuentros donde hay una responsabilidad para mí que mi singularidad empieza a importar, que mi singularidad se pone "en juego" y que estoy en juego. Aquí singularidad no es una cuestión de diferencia - una perspectiva en tercera persona- sino un asunto de irremplazabilidad -en una perspectiva de primera persona. Singularidad, como dice Levinas, se refiere a hacer "lo que nadie más puede hacer en mi lugar" (Levinas, 1989, p. 202) - No hay, por supuesto, nadie que nos puede obligar a asumir la responsabilidad en que nos encontramos. Al respecto, es importante ver que Levinas no está describiendo la responsabilidad como un deber, como algo que debemos hacer. Tampoco lo ve como un hecho biológico, es decir, como algo que no podemos no hacer. Por el contrario, podríamos decir que, en un sentido bastante extraño la libertad humana también significa que tenemos la posibilidad de abandonar la responsabilidad en la que nos encontramos y esto depende totalmente de cada uno de nosotros individualmente. No podemos asumir la responsabilidad de otro ser humano, ni tampoco podemos forzar a otro ser humano a actuar de una manera particular si, desde luego, respetamos su subjetividad, si, claro está, lo consideramos como sujeto de pleno derecho y no como objeto de nuestras acciones e intenciones (esto tiene importantes implicaciones para la educación, cuestión sobre lo cual volveré más adelante).

Una última cosa que vale la pena mencionar aquí es que la responsabilidad en relación con la cual mi singularidad comienza a importar, siempre y estructuralmente, viene desde el exterior en lugar de ser generada por mí. No se inicia a partir de un sentimiento o una necesidad de ser responsable por otro o de cuidar de otro. La responsabilidad ante la cual mi singularidad empieza a importar y en respuesta a la cual mi subjetividad podría llevarse a cabo, en ese momento en particular, singular, aparece siempre como una interrupción de mi 'inmanencia', una interrupción de mi ser con y para mí mismo. Levinas (1989) describe en algunos casos la subjetividad humana como "el fracturamiento de la inmanencia" (p. 204), o en lenguaje un poco menos "fuerte", como el momento en que "lo Mismo - somnoliento en su identidad" es despertado por el otro (p. 209)—.

Tanto Arendt como Levinas tratan de mostrar cómo nuestra subjetividad no está en nuestras manos, pero mientras que la explicación de Arendt comienza por mis iniciativas y cómo deben ser tomadas por los demás a fin de llegar a ser reales, Levinas muestra cómo la posibilidad para mi subjetividad comienza afuera, y solo entonces se convierte en un "asunto" para mí, solo y exclusivamente para mí. Y el "asunto" es si respondo a la responsabilidad en que me encuentro y "doy cuenta", en ese momento, de mi subjetividad, o si me alejo de ella. Si Arendt nos da una teoría de la forma en que nuestra subjetividad no está en nuestras propias manos -y por lo 
tanto nos proporciona una perspectiva en tercera persona- Levinas nos da algo que se parece más a una fenomenología que se acopla con la cuestión de la subjetividad desde una perspectiva en primera persona, mostrando cómo la subjetividad es, en última instancia, una cuestión para que cada uno de nosotros descifre individualmente. Si bien, como se ha mencionado, la teoría podría ayudar, nunca puede sustituir la cuestión existencial; la teoría nunca puede reemplazar el desafío existencial y a veces puede, en realidad, ser una excusa para no vincularse con él.

\section{El punto medio entre la destrucción del mundo y la autodestrucción}

Si las dos secciones anteriores nos dieron algunas ideas sobre lo que significa existir como sujeto, quiero ahora pasar a la segunda parte de mi tesis sobre de la tarea educativa, la cual sugiere que lo que importa en materia educativa es existir en y con el mundo de una manera adulta. La adultez, como ya he sugerido, tiene que ver con el reconocimiento de lo que he denominado la alteridad y la integridad de qué y quién es el otro. Si este reconocimiento no se debe entender como generosidad de mi parte - donde "permitiría" al mundo existir, lo cual, formulado de esta manera, suena en realidad bastante arroganteentonces, ¿cómo podemos darle sentido a lo que esto significa? Una manera de hacerlo es a través de una discusión de lo que podría suceder cuando -en lenguaje de Arendt- nuestras iniciativas encuentran resistencias.

El encuentro con la resistencia, es decir, el encuentro con el hecho de que algo o alguien se resiste a nuestras iniciativas, es una experiencia tremendamente importante, ya que demuestra que el mundo no es solo una construcción de nuestra mente o nuestros deseos, sino que, verdaderamente, tiene una existencia y, por lo tanto, una integridad propia. La experiencia de la resistencia es, en ese sentido, una experiencia mundana; una experiencia de que estamos en algún lugar, no en cualquier parte. ¿Qué hacemos o qué podríamos hacer cuando nos encontramos con la resistencia? Déjenme explorar tres opciones posibles.

Cuando nuestras iniciativas encuentran resistencia, nuestra primera respuesta podría ser de irritación frente al encuentro con algo que las frustra o bloquea o al menos limita nuestra capacidad para ejecutarlas. Podríamos "culpar" por esto a lo que ofrece resistencia y podríamos tratar de hacer cumplir nuestras intenciones - podríamos decir también "hacer cumplir nuestra voluntad" - sobre todo lo que ofrece resistencia. Esto es en parte lo que hay que hacer para que nuestras iniciativas se conviertan en realidad, para llegar al mundo, pero si vamos demasiado lejos en la aplicación de nuestra voluntad sobre el mundo, se llega a un punto en donde nuestra propia fuerza se vuelve tan fuerte que destruye la (integridad de la) "entidad" que ofrece resistencia. Si pensamos en nuestros encuentros con el mundo material, podemos encontrarnos presionando tan duro que el material que queremos formar se rompe bajo la presión. En este momento terminamos destruyendo lo que ofrece resistencia. Podríamos decir, por tanto, que en este extremo del espectro del encuentro con la resistencia existe el riesgo de la destrucción del mundo.

A partir de aquí podemos empezar a ver lo que se encuentra en el otro extremo de este espectro, ya que una segunda manera de responder a la experiencia de la resistencia y, más concretamente, a la frustración de esta experiencia, es retirarse de lo que ofrece resistencia, alejarse de ella. Es aquí cuando decimos que la situación es demasiado compleja y demasiado difícil, que no tenemos la energía o las ganas para perseverar, y por lo tanto, nos retiramos del encuentro con lo que ofrece resistencia: nos echamos para atrás. Aunque pueda haber buenas razones para ello -como dejar espacio para que lo que ofrece resistencia pueda existir y estar en el mundo-el riesgo aquí es que nos apartemos de cualquier compromiso con el mundo, y finalmente, nos retiremos por completo de (la existencia en) el mundo. En una línea similar podría decirse, por tanto, que nos damos por vencidos o que destruimos las mismas condiciones que permiten que podamos existir en el mundo. De ahí que el extremo que aquí encontramos es el riesgo de la autodestrucción.

La destrucción del mundo y la autodestrucción son las respuestas extremas a nuestro encuentro con la resistencia, a nuestro encuentro con el mundo; respuestas en las que, en realidad, terminamos fuera del mundo, en un lugar de no existencia. Ellas también marcan el término medio donde la existencia - la existencia mundana, la existencia en y con el mundo- es posible y, literalmente, tiene lugar. Podríamos referirnos a este término medio como un diálogo, siempre y cuando no pensemos el diálogo como una conversación, sino como una forma de existencia, una manera de estar juntos, que busca hacer justicia a todas las partes involucradas. El diálogo es, en este sentido, fundamentalmente diferente de un debate. Un debate es una forma existencial destinada a producir ganadores y perdedores. Y mientras un debate llega a su fin una vez que alguien ha ganado, el diálogo es un desafío permanente. Una Aufgabe 
permanente, sin fin, podríamos decir. Un debate requiere una explosión de energía; permanecer en el diálogo requiere energía continua y sostenida, atención y compromiso.

El término medio no es un lugar fácil para estar, lo cual ayuda a entender la atracción que ejercen los extremos de la destrucción del mundo y la autodestrucción, pues proporcionan escapes a la dificultad de existir en y con el mundo. A veces necesitamos retirarnos del difícil punto medio para recargar las baterías 0 , tal vez, para tener una perspectiva de lo que encontraremos allí. Y algunas veces es necesario "empujar" hacia algo mejor, lo que también muestra que el término medio no es el espacio donde cualquier cosa deba existir. Pero, en última instancia, es únicamente en el punto medio que la existencia es posible. El término medio, por lo tanto, no es un lugar de pura auto-expresión, sino más bien un lugar donde nuestra auto-expresión se encuentra con barreras, interrupciones, respuestas $-\mathrm{y}$ todas ellas tienen en común la frustración de la que Arendt habla, o la fractura de la inmanencia, a la cual se refiere Levinas. Pero con Levinas podemos decir, también, que estas experiencias nos despiertan de nuestro estado somnoliento de estar fuera del mundo, de estar solo con nosotros mismos. Estas experiencias nos dicen que estamos en ellas "de verdad" - donde lo que hago importa, donde cómo estoy importa y donde yo importo. Mantenerse en el punto medio requiere, de este modo, que afirmemos y tal vez, incluso, abracemos esta dificultad como la dificultad misma que hace posible nuestra existencia. Permanecer en el punto medio requiere tanto el deseo de una existencia en el mundo, una existencia fuera de nosotros mismos - ek-sistencia. Y la tarea educativa, como he sugerido, es despertar un deseo tal en otro ser humano.

\section{Adultez, lo deseado y lo deseable}

Teniendo en cuenta lo que he descrito como adultez en el principio de este artículo, es decir, no como el resultado de un proceso de desarrollo, sino como una cualidad o calidad de la existencia, es posible que tal vez no sea demasiado difícil ver que el término medio entre la destrucción del mundo y la autodestrucción es el lugar donde podría lograrse una forma adulta de ser con lo que es y con quién es el otro. Al referirme a la adultez como algo que podría lograrse estoy subrayando una vez más la fuerza existencial de la línea de pensamiento que estoy presentando en este artículo. Esto significa que la adultez no ha de entenderse como algo que podemos poseer o pretender tener. Tampoco es algo que podamos pretender ser, si por tal afirmación nos referimos a que está en nuestro poder de forma segura y que impregnaría todo lo que hacemos. Lograr llevar a cabo una forma adulta de estar con lo que es y con quien es el otro, es algo que siempre está en juego y es siempre un problema. En cada nueva situación podemos, después de todo, dejar de comportarnos de una manera adulta, y si bien es importante seguir haciendo un esfuerzo - es decir, si deseamos existir en lugar de estar con nosotros mismos- nunca podremos estar seguros del resultado de nuestros esfuerzos, e incluso podemos ser sorprendidos (y decepcionados) por nuestras propias acciones y respuestas.

Si bien la adultez no es un término ideal - pues, como he argumentado, no se refiere a los procesos de crecimiento y, por tanto, a la idea de que es el resultado de dicho proceso-, sí hace referencia a un estado en el que hemos logrado superar otra forma de actuar y responder a lo que es y quien es el otro; una manera que he caracterizado como no adulta, pero que también podríamos llamar "infantil" (ver más adelante), egocéntrica, o con un término sugerido por Levinas, "egológica”, ${ }^{3}$ es decir, siguiendo la lógica del ego, no la lógica de qué y quién es el otro. La forma egológica del ser es generada en su totalidad por los deseos del ego, sin preguntar - y esta es la distinción crucial, cómo y en qué medida tales deseos son deseables, tanto para la existencia del ego en y con el mundo y para el mundo en y con el cual el ego busca existir-.

Esto significa, y es importante, que la adultez no es una supresión de los deseos, sino un proceso a través del cual nuestros deseos reciben una comprobación de la realidad, por así decirlo, cuestionándonos si lo que deseamos es deseable para nuestras propias vidas y las vidas que vivimos con los demás. Tal cuestionamiento - y esto es importante en términos educativos-siempre se plantea como una interrupción de nuestros deseos. Dicha interrupción se manifiesta, en parte, a través de la experiencia de resistencia, e incluso podríamos decir, que cuando nos encontramos con la resistencia no solo encontramos el mundo, sino que al mismo tiempo nos encontramos con los deseos que tenemos en relación con el mundo.

Cuando encontramos resistencia podríamos decir que el mundo está tratando de decirnos algo - y tal vez incluso podríamos decir que el mundo está tratando de enseñarnos algo-. Pero la interrupción también puede realizarse activamente cuando alguien nos pregunta si lo que deseamos es realmente deseable. Y, por supuesto, también podemos llegar a una situación en la que esta pregunta se convierte en

3 En la traducción al Inglés el término que utiliza Levinas es "egology" (Levinas, 1969, p. 35) 
nuestra propia pregunta, en una pregunta que llega a ser vital en nuestra propia vida (vuelvo a la importancia educativa de esto más adelante.) En todo esto, por lo tanto, la ambición no es erradicar nuestros deseos, sino darles una forma y una calidad mundana para que puedan apoyar y sostener una forma adulta de estar en y con el mundo. Spivak $(2004$, p. 526) utiliza la expresión interesante de un "reordenamiento no coercitivo de los deseos" y da esta como su definición de lo que es y de lo que trata la educación.

Con el uso de la oposición entre "adulto" e "infante" no estoy tratando de dar mala fama a los niños o de asumir que todos los adultos son capaces de existir en una forma adulta. Por el contrario, al entender lo infantil y lo adulto, lo egológico y lo no egológico como dos formas diferentes en las que podemos comprometernos con lo que es y con quien es el otro, mi objetivo es hacer visible que ambas opciones están abiertas para niños y para adultos -y tal vez deberíamos decir que solo podemos saber retrospectivamente si hemos salido en una situación particular como niños o como adultos-. Nuestra edad o el tamaño de nuestro cuerpo no son indicadores seguros para ello.

Una última observación que hay que hacer aquí es que si bien debemos prestar atención a lo que cada uno de nosotros tiene que hacer, no debemos olvidar que los entornos en los que actuamos y vivimos también envían mensajes fuertes e influyentes. En la medida en que la vida moderna está estructurada por la lógica del capitalismo, podríamos decir que vivimos en un ambiente que, precisamente, no está interesado en interrumpir y limitar nuestros deseos, sino que, por el contrario, se enfoca hacia su multiplicación, para que deseemos más y, por lo tanto, compremos más y más. Tal "sociedad del impulso" (Roberts, 2014) no está precisamente interesada en nuestra adultez sino que más bien prefiere que nos mantegamos en un estado infantil, ya que es así que está haciendo dinero.

\section{El trabajo educativo: interrupción, suspensión y sustento}

Si esto nos ha traído lo suficiente, aunque no completamente cerca de una comprensión de lo que podría significar existir en y con el mundo de una manera adulta, es decir, como un sujeto, quiero ahora decir algunas cosas sobre el particular trabajo educativo ${ }^{4}$ que podría contribuir a hacer posible tal existencia.

4 Uso la palabra "trabajo" aquí en un sentido muy general, sin pretensiones teóricas particulares.
Tal vez el punto más importante a destacar, particularmente en respuesta a las ideas de moda de que la educación tiene que ver con la promoción del desarrollo del niño, con ayudar a los estudiantes a desarrollar todos sus talentos y alcanzar su pleno potencial, es que el principio fundamental de la educación dirigida a hacer posible una existencia adulta en y con el mundo, es el de la interrupción. Esta línea sigue, en parte, las reflexiones de Arendt sobre la condición de ser sujeto ya que ponen de relieve que nuestra subjetividad no está en nuestras manos, no obstante, sigue, de manera más explícita, la línea de pensamiento trazada por Levinas y su sugerencia de que el acontecimiento de mi subjetividad aparece siempre como una interrupción de mi inmanencia, el ser con y para mí mismo, y como un despertar de este estado de somnolencia.

Mientras que las formulaciones de Levinas pueden parecer abstractas, la idea principal aquí es relativamente simple si vemos, por ejemplo, que todos tenemos talentos para el bien y para el mal, y que tanto la moralidad y la criminalidad se puede entender como los resultados de los procesos de desarrollo. Esto muestra, inmediatamente, que la tarea educativa no puede ser solo la promoción del desarrollo del niño, sino que tiene que preocuparse por la cuestión de qué desarrollo es deseable y cuál no lo es - lo que significa que el gesto fundamental de la educación es el de interrumpir y cuestionar el desarrollo-. También muestra que la tarea educativa nunca puede consistir simplemente en dejar que los estudiantes desarrollen todos sus talentos y alcancen su máximo potencial porque, de nuevo, tras lo que deberíamos estar es el cuestionamiento de los talentos y capacidades con el fin de explorar qué talentos van a ayudar y cuáles van a obstaculizar formas adultas de estar en el mundo - lo que necesariamente requiere de una interrupción en lugar de limitarse a dejar que todo surga, crezca, fluya y florezca-. Sugerir que la educación solo consiste en apoyar el desarrollo del niño, a punto de dejar que cada estudiante desarrolle sus talentos y alcance su máximo potencial, por lo tanto, es una mentira - una mentira que no solo es engañosa hacia los niños y estudiantes, sino que también es engañosa como vocabulario para que los educadores describan e incluso entiendan cuál es su tarea en realidad-

Mientras que la interrupción es, en este sentido, el término más importante, ya que pone de relieve la estructura fundamental de la labor educativa, es importante ver que las interrupciones pueden llevarse a cabo de maneras diferentes - algunas de ellas educativas (orientadas a potenciar la adultez) y otras no-. Una forma no educativa de realizar la 
interrupción es aquella que podríamos llamar educación moral directa, donde la interrupción se establece como un juicio directo por parte del educador sobre el niño, es decir, en forma de condena - "iincorrecto!"- o elogio - "correcto"- El problema aquí no es la retroalimentación en sí misma, lo cual es importante y, hasta cierto grado útil e importante, sino el hecho de que el juicio viene del educador y se aplica al niño, ya que esto no le da al niño ni tiempo ni oportunidad de aparecer como sujeto en relación con tal juicio. El niño no es más que un objeto del juicio del educador (o, para hacer un pequeño juego de palabras, el niño queda sujeto al juicio del educador).

También podemos poner esto en términos de la distinción entre lo deseado y lo deseable. Esta distinción, como he sugerido, marca la diferencia entre una forma infantil y una adulta de existir, sin embargo, es importante leer esto con cuidado, ya que la idea no es que los "deseos" son la forma infantil y lo "deseable" la forma adulta. Es más bien que la forma adulta se caracteriza por la "capacidad" - pero, tal vez, deberíamos llamarla disposición o deseo en sí mismo- de hacer y reflexionar sobre la distinción entre los propios deseos y su posible conveniencia. La diferencia, en otras palabras, es entre ser un objeto de los propios deseos -o en una formulación más precisa: estar sujeto a los propios deseos-y ser sujeto de los propios deseos.

Mientras el educador decida por el niño o estudiante cuáles de sus deseos son deseables, el niño y el estudiante siguen siendo objetos de sus intenciones $\mathrm{y}$ actividades. El reto educativo clave, por tanto, no es simplemente decirle al niño o estudiante cuáles de sus deseos son deseables, sino que esta cuestión se convierta en una cuestión vital para el niño o estudiante. Esto requiere cualquier cosa menos una educación moral directa; más bien apunta a la necesidad de abrir espacios literales y metafóricos donde el niño o el estudiante pueda establecer una relación con sus deseos, así como también requiere la creación de una brecha entre los deseos que puedan surgir y las acciones que se derivan de ellos. El principio educativo aquí es el de suspensión - suspensión en el tiempo y espacio, podría decirse-que proporciona oportunidades para establecer relaciones con nuestros deseos, hacerlos visibles, perceptibles, para que podamos trabajar en ellos. ${ }^{5}$ Esto, para decirlo una vez más, no es un proceso en el cual superamos o destruimos nuestros deseos - nuestros deseos son, des-

5 En Biesta (2017) discuto con mayor detalle la contribución especial que las artes pueden hacer en este proceso. pués de todo, una fuerza impulsora fundamentalsino que seleccionamos y transformamos nuestros deseos para que pasemos de estar sujetados a ellos a convertirnos en sujetos de nuestros deseos. Este es el "reordenamiento no coercitivo de los deseos" sobre el que Spivak (2004, p. 526) escribe - lo cual es, quizás, un poco más coercitivo de lo que su formulación sugiere $-y$, además del reordenamiento, tambiém puede implicar un cambio en la intensidad de nuestros deseos.

Tanto la interrupción como la suspensión tienen lugar en el punto medio con el propósito de mantener allí al estudiante, ya que es solamente allí donde la adultez puede alcanzarse. Esto significa que una tercera dimensión de la labor educativa - y tal vez la dimensión más importante y más precaria- es la de apoyar al estudiante para que permanezca en el difícil punto medio. Se trata de proporcionar sustento, en cualquier forma imaginable, de modo que el estudiante pueda soportar la dificultad de existir en y con el mundo. Sin embargo, como el punto medio es el lugar donde el estudiante se encuentra con el mundo, parte del trabajo educativo es, también, hacer posible este encuentro y darle forma - cosa que tiene que ver con la pedagogía y el currículo-. Más concretamente, la tarea consiste en dar forma a la experiencia de la resistencia, de modo que haya una posibilidad real de experimentar el mundo en su alteridad y la integridad. Por lo tanto, también significa proporcionar tiempo para encontrar la experiencia de la resistencia y trabajar con ella- o con una expresión bien interesante: para trabajar $a$ través de ella-.

El trabajo del educador es, también, "poner en escena" la experiencia de la resistencia como importante, significativa y positiva y tener buen ojo para las diferentes maneras sutiles en que esto se puede hacer. No se trata de hacer las cosas difíciles para dificultarlas, sino para reconocer su importancia fundamental en relación con la cuestión de estar en el mundo como sujeto. Visto de esta manera, es una advertencia contra la supresión de toda resistencia en la educación, haciéndola flexible, personalizada y completamente adaptada a las necesidades individuales de cada niño o estudiante. Dichas estrategias corren el riesgo de aislar al estudiante del mundo en lugar de apoyar al estudiante en el diálogo con el mundo. Mostrar al alumno dónde y cómo el encuentro con la experiencia de la resistencia es educativamente significativa - que no es simplemente decirle que esto es así, sino que puede adoptar muchas formas diferentes-es importante con el fin de mantener al niño alejado de 
los dos extremos de la destrucción del mundo y la autodestrucción. 0 , en lugar de utilizar la expresión "negativa" de "mantenerlo alejado", el trabajo del educador es despertar el deseo en el estudiante de querer permanecer en el difícil punto medio.

\section{Transformando el poder en autoridad: el hermoso riesgo de enseñar}

Si la interrupción, suspensión y sustento son, en cierto sentido, actividades más bien concretas, hay una dimensión adicional de la obra del maestro que apenas está en sus manos: se trata de la transformación del poder en autoridad. El problema aquí es que a pesar de que las interrupciones educativas están "dirigidas" a la subjetividad del estudiante, cuando se llevan a cabo aparecen como actos de poder, al menos en aquellos casos en los que los estudiantes no pidieron que tales interrupciones se produjeran, que es probablemente donde toda la educación comienza. El gran propósito de las interrupciones educativas es "dirigir" a los estudiantes hacia la pregunta de si lo que desean es lo que deberían desear, y gran parte del trabajo del educador consiste en crear el tiempo, el espacio y las formas para que los estudiantes puedan encontrar sus deseos, examinarlos, seleccionarlos y transformarlos. Mientras que la cuestión de lo deseado y lo deseable es introducida por el maestro - como una potente interrupción - la ambición es que esta cuestión se convierta en un asunto vital para el estudiante. Lo que aquí está en juego es la cuestión de qué debería tener autoridad en nuestras vidas y la cuestión de la autoridad es, precisamente, entrar en diálogo con qué y quién es el otro. Se trata de dejar que algo o alguien tenga poder en nuestras vidas. Se trata de autorizar qué y quién es el otro, de dejar que hable, por así decirlo, de convertirlo en un autor.

Lo que esperamos como maestros cuya enseñanza está dirigida hacia la subjetividad de nuestros estudiantes es que en algún momento el estudiante se vuelva hacia nosotros y reconozca que lo que en un primer momento le pareció como una interrupción no deseada - un acto de poder- en realidad contribuyó a su existencia adulta en y con el mundo, su subjetividad adulta. Cuando ocurre un giro de este tipo, y solo cuando ocurre, podemos decir que el poder -que siempre es monológico y unidireccional- se ha transformado en autoridad -que siempre es dialógica y relacional (Bingham, 2008)-. Pero nunca sabremos si tal "viraje" puede ocurrir o cuando puede ocurrir, pues bien puede ser mucho tiempo después de que el estudiante haya desaparecido de nuestra vista y nuestra vida (profesional). Esto significa que cualquier enseñanza dirigida hacia la subjetividad del estudiante es, ante todo, arriesgada, pues sus resultados son impredecibles. Pero también es arriesgada porque, como maestros, nos arriesgamos a nosotros mismos, ya que siempre estamos representando el poder sin saber si este poder "retorna" como autoridad, como poder aceptado y reconocido. No obstante, esto no debería impedirnos asumir ese riesgo, ya que sin él tampoco se produciría la educación. Más bien, debería ayudarnos a entender mucho mejor cuál es la naturaleza arriesgada de la enseñanza y de la educación en general; si se dirige hacia la subjetividad del estudiante, si apunta a despertar en otro ser humano el deseo por querer existir en el mundo de una manera adulta.

\section{Referencias}

Andreotti, V. (2011). Actionable postcolonial theory in education. Nueva York, NY: Palgrave/Macmillan.

Arendt, H. (1958). The human condition. Chicago, IL: The University of Chicago Press.

Bauman, Z. (1993). Postmodern ethics. Oxford, Reino Unido: Wiley-Blackwell.

Biesta, G. (2006). Beyond learning. Democratic education for a human future. Boulder, CO: Paradigm Publishers.

Biesta, G. (2010a). A new "logic" of emancipation: The methodology of Jacques Ranciere. Educational Theory 60(1), 39-59.

Biesta, G. (2010b). How to exist politically and learn from it: Hannah Arendt and the problem of democratic education. Teachers College Record, 112(2), 558-577.

Biesta, G. (2017). Letting art teach: Art education after Joseph Beuys. Arnhem: ArtEZ Press.

Biesta, G. \& Säfström, C. (2011). A manifesto for education. Policy Futures in Education 9(5), 540-547.

Bingham, C. (2008). Authority is relational. Albany, NY: SUNY Press.

Critchley, S. (1999). Ethics, politics, subjectivity. Londres/ Nueva York: Verso.

Levinas, E. (1969). Totality and infinity. An essay on exteriority. Pittsburgh, PA \& The Hague: Duquesne University Press \& Martinus Nijhoff. (Obra publicada originalmente en1961). 
Levinas, E. (1985). Ethics and infinity. Conversations with Philippe Nemo. Pittsburgh, PA: Duquesne University Press.

Levinas, E. (1989). Revelation in the Jewish tradition. En S. Hand (Ed.), The Levinas reader (pp.190-211). Oxford, UK: Blackwell.

Lingis, A. (1994). The community of those who have nothing in common. Bloomington, IN: Indiana University Press.

Meirieu, P. (2007). Pédagogie: Le devoir de résister. Issy-lesMoulineaux: ESF éditeur.

Roberts, P. (2014). The impulse society. What is wrong with getting what we want. Londres, Reino Unido: Bloomsbury.
Sartre, J. (2007[1946]). Existentialism is a humanism. New Haven, CT: Yale University Press. (Obra publicada originalmente en 1988).

Spivak, G. (1988). Can the subaltern speak? En C. Nelson \& L. Grossberg (Eds.), Marxism and the interpretation of culture (pp. 271-313). Urbana, IL: University of Illinois Press.

Spivak, G. (2004). Righting the wrongs. South Atlantic Quarterly, 103(2/3), 523-581. 